\title{
Systematic Analysis of Accuracy in Predicting Complete Oncological Resection in Pancreatic Cancer Patients-Proposal of a New Simplified Borderline Resectability Definition
}

\author{
Louisa Bolm ${ }^{1}$ (D), Katharina Mueller ${ }^{1}$, Katharina May ${ }^{2}$, Stefan Sondermann ${ }^{3}$ (D), \\ Ekaterina Petrova ${ }^{1}$, Hryhoriy Lapshyn ${ }^{1}$, Kim Christin Honselmann ${ }^{1}$, Dirk Bausch ${ }^{4}$, \\ Sergii Zemskov ${ }^{5}$, Peter Bronsert ${ }^{6,7}$ (D), Tobias Keck ${ }^{1, *}$, Steffen Deichmann ${ }^{1}$ (D) and \\ Ulrich F. Wellner ${ }^{1}$ (D)
}

1 Department of Surgery, University Medical Center Schleswig-Holstein, Campus Luebeck, 23562 Luebeck, Germany; louisa.bolm@googlemail.com (L.B.); katharina.mueller@uksh.de (K.M.); ekaterina.petrova@uksh.de (E.P.); hryhoriy.lapshyn@uksh.de (H.L.); kim.honselmann@uksh.de (K.C.H.); steffen.deichmann@uksh.de (S.D.); ulrich.wellner@uksh.de (U.F.W.)

2 Department of Radiology, University Medical Center Schleswig-Holstein, Campus Luebeck, 23562 Luebeck, Germany; katharina.may@uksh.de

3 Department of Neuroradiology, University Medical Center Schleswig-Holstein, Campus Luebeck, 23562 Luebeck, Germany; Stefan.Sondermann@uksh.de

4 Department of Surgery, Marien Hospital, University Hospital of Ruhr University Bochum, 44621 Herne, Germany; dirk.bausch@dbausch.de

5 Department of General Surgery, Bogomolets National Medical Unoversity, 01601 Kiev, Ukraine; szemskovmeister@gmail.com

6 Department of Pathology, Medical Center-University of Freiburg, Faculty of Medicine, University of Freiburg, 79106 Freiburg, Germany; peter.bronsert@uniklinik-freiburg.de

7 German Cancer Consortium (DKTK), Partner Site Freiburg, 79106 Freiburg, Germany

* Correspondence: Tobias.Keck@uksh.de; Tel.: +49-451-500-40100; Fax: +49-451-500-40104

Received: 26 February 2020; Accepted: 1 April 2020; Published: 4 April 2020

\begin{abstract}
Background: Borderline resectability in pancreatic cancer (PDAC) is currently debated. Methods: Patients undergoing pancreatic resections for PDAC were identified from a prospectively maintained database. As new borderline criteria, the presence of any superior mesenterico-portal vein alteration (SMPV) and perivascular stranding of the superior mesenteric artery (SMA) was evaluated in preoperative imaging. The accuracy of established radiological borderline criteria as compared to the new borderline criteria in predicting $\mathrm{R}$ status (sensitivity/negative predictive value) and overall survival was assessed. Results: 118 patients undergoing pancreatic resections for PDAC from 2013 to 2018 were identified. Forty-three (36.4\%) had radiological perivascular SMA stranding and 55 (46.6\%) had SMPV alterations. Interrater reliability was 90\% for SMA stranding and 87\% for SMPV alterations. The new borderline definition including SMPV alterations and perivascular SMA stranding was the best predictor of conventional R status ( $p=0.040$, sensitivity 53\%, negative predictive value $81 \%$ ) and Leeds/Wittekind circumferential margin status ( $p=0.050$, sensitivity $73 \%$, negative predictive value $79 \%$ ) as compared to established borderline resectability definition criteria. Perivascular SMA stranding qualified as an independent negative prognostic parameter (HR 3.066, 95\% CI 1.078-5.716, $p=0.036)$. Conclusion: The radiological evaluation of any SMPV alteration and perivascular SMA stranding predicts $R$ status and overall survival in PDAC patients, and may serve to identify potential candidates for neoadjuvant therapy.
\end{abstract}

Keywords: pancreatic cancer; oncological resection; borderline resectability 


\section{Introduction}

Pancreatic ductal adenocarcinoma (PDAC) is an aggressive solid malignancy associated with poor prognosis $[1,2]$. The majority of patients present with distant metastases at the time of diagnosis, and less than $50 \%$ of all PDAC patients are eligible for upfront curative resection [3].

Over the past years, attempts were made to specify resectability criteria in pancreatic cancer patients. While irresectability is well-defined, the definition of borderline resectability is currently debated. Borderline resectable PDAC tumors are technically resectable at a high risk of margin positive resection [4]. In 2014, the International Study Group of Pancreatic Surgery (ISGPS) aimed to standardize the definition and approach to borderline resectable PDAC [5]. The criteria of borderline resectability were adopted from the National Comprehensive Cancer Network (NCCN) definition, comprising the absence of distant metastases, the involvement of the superior mesenterico-portal vein (SMPV) and tumor abutment of a maximum of $180^{\circ}$ in the superior mesenteric artery (SMA). The 2014 ISGPS definition relies on anatomic criteria evaluated preoperatively. As a major obstacle, these criteria cannot always be safely determined in preoperative imaging. Therefore, intraoperative re-evaluation is necessary and may often reveal criteria of irresectability.

In 2017, the International Association of Pancreatology (IAP) added new dimensions to the definition of borderline resectability [6]. In addition to anatomical criteria, aspects of tumor biology in terms of elevated serum CA19-9 levels and patient performance status were defined as further determinants of borderline resectability. Anatomical criteria mainly regarding the involvement of the SMPV were refined by further amendments. By adding these additional aspects, the definition of borderline resectability has become increasingly complex, and translation to clinical practice remains challenging.

The main aim in borderline resectable PDAC is to safely achieve margin negative resection. Despite updated borderline resectability definitions, preoperative parameters predicting R0 resections in borderline resectable PDAC patients are lacking. The accuracy of $\mathrm{R}$ status prediction by distinct borderline resectability definitions has not been assessed systematically yet. Mainly in the light of neoadjuvant therapy, the preoperative imaging-based prediction of $\mathrm{R}$ status is of major importance. Those PDAC patients with a high risk of margin positive resections should be considered for neoadjuvant therapy.

The accuracy of established borderline criteria in predicting $\mathrm{R}$ status is unclear, and their applicability to clinical routine appears limited due to complex scoring systems. We intended to develop simplified preoperative radiological criteria safely predicting $R$ status. The aim of this study was to systematically assess the prediction of $\mathrm{R}$ status, comparing established borderline resectability criteria and novel radiological parameters by the means of a diagnostic accuracy study. Our hypothesis is that a simplified borderline resectability scoring system would improve both the quality of $\mathrm{R}$ status prediction and the willingness of radiologists and surgeons to integrate the scoring system into clinical routine.

\section{Results}

\subsection{Baseline Parameters and Perivascular SMA Stranding}

A total of 118 patients undergoing pancreatoduodenectomy (PD) for PDAC in the period from 2013 to 2018 were identified, and the median follow-up was 12 months. The median age at operation was 68 years, ranging from 41 to 85 . The median preoperative serum CA19-9 level was $121 \mathrm{IU} / \mathrm{mL}$, ranging from 0.06 to $2945 \mathrm{IU} / \mathrm{mL}$. According to the ISGPS 2014 definition, 50 patients $(42.4 \%)$ were classified as borderline resectable. According to the IAP 2017 definition, 88 patients $(74.6 \%)$ were considered borderline resectable. Sixty-one patients (51.7\%) underwent a pylorus-preserving pancreatoduodenectomy (PPPD), 16 patients (13.6\%) had a Whipple procedure, 19 patients $(16.1 \%)$ received a distal pancreatectomy and 22 patients $(18.6 \%)$ had a total pancreatectomy. A total of 38 $(32.2 \%)$ patients underwent portal vein resections (PVRs). 
In a pilot study, the interrater reliability for the novel borderline criteria (SMA stranding and SMPV alterations) was assessed for two surgeons and two radiologists blinded for outcome variables. The raters evaluated SMA stranding and SMPV alterations as either positive or negative in 30 PDAC patients based on preoperative CT-based imaging. The interrater reliability was 90\% $($ Kappa = 0.77) for SMA stranding and 87\% (Kappa 0.75) for SMPV alterations.

For the study cohort of 118 patients, the novel borderline resectability criteria were assessed by an independent radiologist and two independent trained surgeons blinded for patient outcome. A total of 43 patients $(36.4 \%)$ had positive perivascular SMA stranding. Fifty-three patients $(54.9 \%)$ had at least one SMPV alteration. Patients with SMA stranding were more often diagnosed as borderline resectable according to the ISGPS 2014 criteria $(30.7 \%$ vs. $62.8 \%, p=0.001)$ and according to the IAP 2017 definition $(65.3 \%$ vs. 90.7\%, $p=0.002)$. Furthermore, SMPV alterations were more frequent in patients with SMA stranding $(67.4 \%$ vs. $32.0 \%, p=0.001)$. Positive N status $(81.4 \%$ vs. $61.3 \%, p=0.025)$ and perineural invasion $(90.5 \%$ vs. $73.3 \%, p=0.032)$ were more often detected in patients with SMA stranding. Differences in baseline parameters between patients with SMA stranding vs. no stranding are displayed in Table 1.

Table 1. Baseline parameters in patients with superior mesenteric artery (SMA) stranding versus no SMA stranding.

\begin{tabular}{|c|c|c|c|}
\hline Baseline Parameters SMA Stranding & No SMA Stranding & Positive SMA Stranding & \\
\hline Total $n$ & 75 & 43 & \\
\hline Parameter & $n(\%) /$ median (range) & $n(\%) /$ median (range) & $p$-value \\
\hline \multicolumn{4}{|l|}{ Age } \\
\hline$<68$ & $39(52.0)$ & $20(46.5)$ & \\
\hline$>68$ & $36(48.0)$ & $23(43.5)$ & 0.702 \\
\hline \multicolumn{4}{|l|}{ Sex } \\
\hline male & $31(41.3)$ & $26(60.5)$ & \\
\hline female & $44(58.7)$ & $17(39.5)$ & 0.060 \\
\hline \multicolumn{4}{|l|}{ ASA Score } \\
\hline $0-2$ & $38(50.7)$ & $15(34.9)$ & \\
\hline $3-4$ & $37(49.3)$ & $28(65.1)$ & 0.125 \\
\hline \multicolumn{4}{|l|}{ Serum CA 19-9 } \\
\hline$>40 \mathrm{U} / \mathrm{L}$ & $22(66.7)$ & $20(80.0)$ & 0.375 \\
\hline \multicolumn{4}{|l|}{ Borderline ISGPS 2014 Definition } \\
\hline BR- & $52(69.3)$ & $16(37.2)$ & \\
\hline $\mathrm{BR}+$ & $23(30.7)$ & $27(62.8)$ & 0.001 \\
\hline \multicolumn{4}{|l|}{ Borderline IAP 2017 Definition } \\
\hline BR- & $26(34.7)$ & $4(9.3)$ & \\
\hline $\mathrm{BR}+$ & $49(65.3)$ & $39(90.7)$ & 0.002 \\
\hline \multicolumn{4}{|l|}{ Any SMPV alterations } \\
\hline no & $51(68.0)$ & $14(32.6)$ & \\
\hline yes & $24(32.0)$ & $29(67.4)$ & 0.001 \\
\hline \multicolumn{4}{|l|}{ PVR } \\
\hline no & $50(66.7)$ & $30(69.8)$ & \\
\hline yes & $25(33.3)$ & $13(30.2)$ & 0.839 \\
\hline \multicolumn{4}{|l|}{ Multivisceral resection } \\
\hline no & $70(93.3)$ & $38(88.4)$ & \\
\hline yes & $5(6.7)$ & 5 (11.6) & 0.494 \\
\hline \multicolumn{4}{|l|}{ T stage } \\
\hline $\mathrm{T} 1 / 2$ & $17(22.7)$ & $10(23.3)$ & \\
\hline T3/4 & $58(77.3)$ & 33 (76.7) & 1.000 \\
\hline \multicolumn{4}{|l|}{ N stage } \\
\hline No & $29(38.7)$ & 8 (18.6) & \\
\hline $\mathrm{N}+$ & $46(61.3)$ & 35 (81.4) & 0.025 \\
\hline \multicolumn{4}{|l|}{$\mathbf{M}$} \\
\hline M0 & $72(96.0)$ & $41(95.3)$ & \\
\hline M1 & $3(4.0)$ & $2(4.7)$ & 1.000 \\
\hline
\end{tabular}


Table 1. Cont.

\begin{tabular}{cccc}
\hline Baseline Parameters SMA Stranding & No SMA Stranding & Positive SMA Stranding & \\
\hline LNR & & & \\
$<$ median 0.08 & $43(57.3)$ & $17(39.5)$ & 0.085 \\
$>$ median 0.08 & $32(42.7)$ & $26(60.5)$ & \\
Grading & & & \\
G 1/2 & $53(71.6)$ & $32(84.2)$ & \\
G 3/4 & $21(28.4)$ & $6(15.8)$ & 1.000 \\
L0 & $45(60.0)$ & $26(60.5)$ & \\
L1 & $30(40.0)$ & $17(39.5)$ & \\
Lymphovascular invasion & $59(78.7)$ & $28(65.1)$ & \\
V0 & $16(21.3)$ & $15(34.9)$ & \\
V1 & $20(26.7)$ & $4(9.5)$ & 0.130 \\
Pascular invasion & $55(73.3)$ & $38(90.5)$ & \\
Pn1 & $35(46.7)$ & $26(60.5)$ & 0.182 \\
Perineural invasion & $40(53.3)$ & $17(39.5)$ & \\
no & & & \\
yes & & & \\
\hline
\end{tabular}

SMA: superior mesenteric artery; BMI: body mass index; ASA score: American Society of Anesthesiologists score; CA19-9: carbohydrate antigen 19-9; ISGPS: International Study Group of Pancreatic Surgery; IAP: International Association of Pancreatology; SMPV: superior mesenterico-portal vein; PVR: portal vein resection; LNR: lymph node ratio.

\subsection{Borderline Resectability Definitions and Accuracy in the Prediction of $R$ Status}

Sensitivity of borderline resectability definition criteria in predicting positive $\mathrm{R}$ status was calculated. Furthermore, the negative predictive value predicting $\mathrm{R} 0$ resection in the absence of positive borderline criteria was calculated. The ISGPS 2014 definition had a low sensitivity and low negative predictive value (NPV) in predicting UICC/AJCC (= conventional) R status (sensitivity 48\%, NPV 64\%) and Leeds/Wittekind R status (sensitivity 70\% and NPV 60\%). The IAP 2017 definition also had a low sensitivity and NPV regarding the prediction of conventional R status (sensitivity 43\%, NPV 31\%) and Leeds/Wittekind R status (sensitivity 69\% and NPV 29\%). SMPV alterations were associated with an improved sensitivity and NPV in predicting conventional R status (sensitivity 45\%, NPV 60\%) and Leeds/Wittekind R status (sensitivity 70\% and NPV 60\%). Perivascular SMA stranding was associated with a high sensitivity and NPV in predicting conventional R status (sensitivity 53\%, NPV 73\%) and Leeds/Wittekind R status (sensitivity $80 \%$ and NPV 76\%). The combination of SMPV alterations and SMA stranding as novel borderline resectability definition criteria was associated with an increased sensitivity and an even higher NPV in predicting conventional $R$ status (sensitivity 53\%, NPV 80\%) and Leeds/Wittekind R status (sensitivity 73\% and NPV 79\%). Further details are displayed in Table 2. Regarding conventional $\mathrm{R}$ status, patients undergoing PVRs were not more likely to undergo R1 resection (SMV resection: 47.4\% R1, versus no SMV resection: 33.8\% R1; $p=0.0163$ ). However, patients with PVRs more often showed R1 resection according to Leeds/Wittekind R status (SMV resection: 81.6\% R1 versus no SMV resection: $61.3 \% \mathrm{R} 1$ ).

Based on the high accuracy of the novel radiological parameters in predicting $\mathrm{R}$ status, a new borderline resectability scoring system was developed. This scoring system divides patients into three major subgroups based on the probability of R1 or R0 resection. Group A is defined as patients with a high probability of R0 resection (80\%); these patients show neither SMA stranding nor SMPV alterations. Patients in group B have an increased risk of R1 resection $(50 \%-80 \%)$ and present with either SMA stranding or SMPV alterations in preoperative imaging. Group $\mathrm{C}$ is defined as patients with a high risk of $\mathrm{R} 1$ resection $(70 \%-80 \%)$, showing both SMA stranding and SMPV alterations. Figure 1 displays an overview of the groups. 
Table 2. Borderline resectability and prediction of $\mathrm{R}$ status.

\begin{tabular}{|c|c|c|c|c|c|c|c|c|}
\hline \multicolumn{9}{|c|}{ Borderline Resectability Definitions and Prediction of $R$ Status } \\
\hline & \multicolumn{8}{|c|}{ UICC/AJCC R Status } \\
\hline & & \multicolumn{2}{|c|}{ R0 } & \multicolumn{2}{|c|}{$\mathbf{R +}$} & \multirow[b]{2}{*}{$p$-value } & \multirow[b]{2}{*}{ Sensitivity } & \multirow[b]{2}{*}{$N P V$} \\
\hline & & $n /$ median & $\% /$ range & $n /$ median & $\% /$ range & & & \\
\hline total $n$ & & 73 & 61.9 & 45 & 38.1 & & & \\
\hline \multirow[t]{2}{*}{ Borderline ISGPS 2014 Definition } & BR- & 47 & 64.4 & 21 & 46.7 & 0.084 & $48 \%$ & $64 \%$ \\
\hline & $\mathrm{BR}+$ & 26 & 35.6 & 24 & 53.3 & & & \\
\hline \multirow[t]{2}{*}{ Borderline IAP 2017 Definition } & BR- & 23 & 31.5 & 7 & 15.6 & 0.081 & $43 \%$ & $31 \%$ \\
\hline & $\mathrm{BR}+$ & 50 & 68.5 & 38 & 84.4 & & & \\
\hline \multirow[t]{2}{*}{ Any SMPV alterations } & no & 44 & 60.3 & 21 & 46.7 & 0.183 & $45 \%$ & $60 \%$ \\
\hline & yes & 29 & 39.7 & 24 & 53.3 & & & \\
\hline \multirow[t]{2}{*}{ SMA Stranding } & no & 53 & 72.6 & 22 & 48.9 & 0.011 & $53 \%$ & $73 \%$ \\
\hline & yes & 20 & 27.4 & 23 & 51.1 & & & \\
\hline \multirow[t]{5}{*}{ Borderline novel Definition } & BR- & 59 & 80.8 & 29 & 64.4 & 0.040 & $53 \%$ & $81 \%$ \\
\hline & $\mathrm{BR}+$ & 14 & 19.2 & 16 & 35.6 & & & \\
\hline & & \multicolumn{7}{|c|}{ Leeds/Wittekind R status } \\
\hline & & \multicolumn{2}{|c|}{ R0 wide } & \multicolumn{2}{|c|}{ R0 narrow/R1 } & & & \\
\hline & & $n /$ median & $\% /$ range & $n /$ median & $\% /$ range & $p$-value & Sensitivity & $N P V$ \\
\hline total $n$ & & 38 & 32.2 & 80 & 67.8 & & & \\
\hline \multirow[t]{2}{*}{ Borderline ISGPS 2014 Definition } & BR- & 23 & 60.5 & 45 & 56.3 & 0.695 & $60 \%$ & $60 \%$ \\
\hline & $\mathrm{BR}+$ & 15 & 39.5 & 35 & 43.7 & & & \\
\hline \multirow[t]{2}{*}{ Borderline IAP 2017 Definition } & BR- & 11 & 28.9 & 19 & 23.8 & 0.652 & $69 \%$ & $29 \%$ \\
\hline & $\mathrm{BR}+$ & 27 & 71.1 & 61 & 76.2 & & & \\
\hline \multirow[t]{2}{*}{ Any SMPV alterations } & no & 23 & 60.5 & 42 & 52.5 & 0.436 & $70 \%$ & $60 \%$ \\
\hline & yes & 15 & 39.5 & 38 & 47.5 & & & \\
\hline \multirow[t]{2}{*}{ SMA Stranding } & no & 29 & 76.3 & 46 & 57.5 & 0.036 & $80 \%$ & $76 \%$ \\
\hline & yes & 9 & 23.7 & 34 & 42.5 & & & \\
\hline \multirow[t]{2}{*}{ Borderline novel Definition } & BR- & 30 & 78.9 & 58 & 72.5 & 0.505 & $73 \%$ & $79 \%$ \\
\hline & $\mathrm{BR}+$ & 8 & 21.1 & 22 & 27.5 & & & \\
\hline
\end{tabular}

SMA: superior mesenteric artery; ISGPS: International Study Group of Pancreatic Surgery; IAP: International Association of Pancreatology; SMPV: superior mesenterico-portal vein; PVR: portal vein resection; LNR: lymph node ratio; BR+: Borderline resectable; BR-: Not borderline resectable.

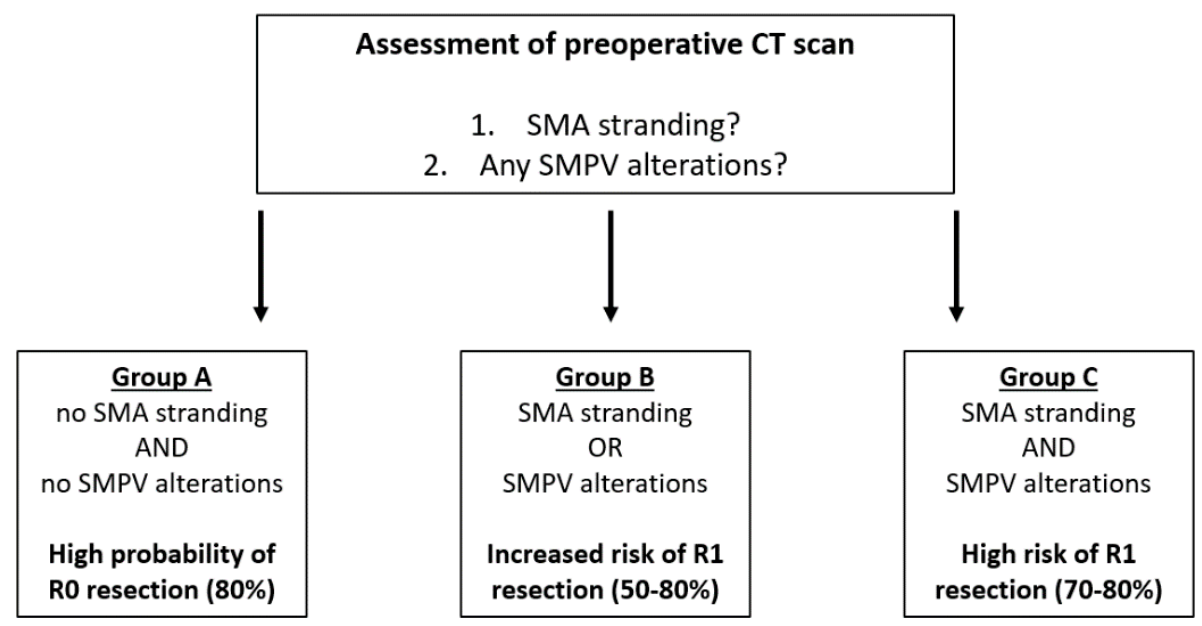

Figure 1. A novel scoring system for borderline resectability. SMA: superior mesenteric artery; SMPV: superior mesenterico-portal vein.

\subsection{Survival Analysis Total Cohort}

Median overall survival (OS) in all patients was 12 months. Prognostic parameters in the univariate analysis were the ASA score (ASA 0-2: 31 months vs. 3-4: 22 months, $p=0.044$ ), the ISGPS 2014 borderline criteria (borderline: 19 months vs. resectable: 30 months, $p=0.030$ ), the IAP 2017 
borderline criteria (borderline: 20 months vs. resectable: 40 months, $p=0.002$ ), SMPV alterations (SMPV alterations: 18 months vs. no SMPV alterations: 31 months, $p=0.017$ ), SMA stranding (positive stranding: 13 months vs. no stranding: 30 months, $p<0.001$ ), the novel borderline definition (borderline: 13 months vs. resectable: 29 months, $p=0.006)$, the operation time (>374 min: 18 months vs. $<374$ min: 30 months, $p=0.003$ ), portal vein resection (PVR 18 months: vs. no PVR: 31 months, $p=0.012$ ), N stage (N0: 33 months vs. $\mathrm{N}+: 20$ months, $p=0.022)$, lymphovascular invasion (L+: 17 months vs. L0: 31 months, $p=0.002)$, vascular invasion (V+: 14 months vs. V0: 30 months, $p=0.001$ ), perineural invasion (Pn+: 22 months vs. Pn0: 40 months, $p=0.023$ ), UICC/AJCC R status (R0: 30 months, vs. R+: 18 months, $p=0.016$ ) and Leeds/Wittekind R status (R0 wide: 34 months vs. R0 narrow/R1: 21 months, $p=0.041)$. For the survival curves of negative versus positive SMA stranding, see Figure 2.

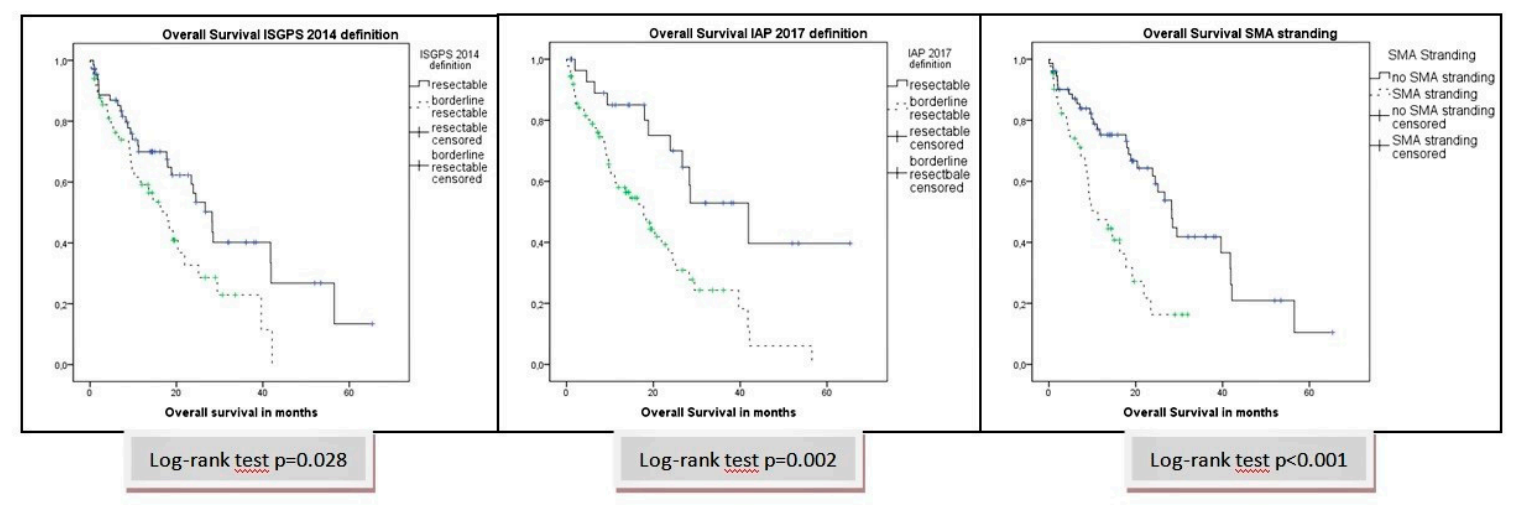

Figure 2. Borderline resectability definitions and overall survival. SMA: superior mesenteric artery; ISGPS: International Study Group of Pancreatic Surgery; IAP: International Association of Pancreatology.

In the multivariate analysis, SMA stranding (HR 3.066, 95\% CI 1.078-5.716, $p=0.036$ ), operation time (HR 1.989, 95\% CI 1.055-3.748, $p=0.044$ ) and vascular invasion (HR 2.375, 95\% CI 1.201-4.700, $p=0.013$ ) remained independent prognostic parameters. Details of the survival analysis are displayed in Supplementary Table S1.

\section{Discussion}

In the current study, a new simplified borderline resectability scoring system was developed based on perivascular stranding of the superior mesenteric artery (SMA) and any alterations of the superior mesenterico-portal vein (SMPV). The scoring system identifies patients with a high probability of $\mathrm{R} 0$ resection (group A), patients with an increased risk of R1 resections (group B) and patients with a high risk of R1 resection (group C). The current study was the first, to the best of our knowledge, to systematically evaluate the accuracy of borderline resectability definitions in predicting $\mathrm{R}$ status. The novel simplified borderline scoring system qualified best to predict conventional $\mathrm{R}$ status by UICC/AJCC R status and Leeds/Wittekind circumferential margin status-better than the ISGPS 2014 and IAP 2017 borderline resectability definitions. Perivascular SMA stranding is associated with perineural invasion and remained an independent prognostic parameter with a negative impact on long-term overall survival. Thus, we developed a scoring system simplifying the diagnostic process for borderline resectable PDAC patients and developed a tool to accurately predict $\mathrm{R}$ status and patient prognosis.

Borderline resectable PDAC is considered resectable with a high risk of macroscopic or microscopic margin positive resection [4]. In 2014, a consensus statement by the international study group of pancreatic surgery (ISGPS) aimed to precisely separate borderline PDAC from irresectable PDAC, and to standardize the definition criteria based on preoperative radiological parameters [5]. While any SMPV occlusion and any arterial encasement had been considered a criterion of irresectability before, the ISGPS followed the National Comprehensive Cancer Network (NCCN) definition of borderline 
resectability and classified SMPV distortion and even short-segment venous occlusion-as well as the encasement of the gastroduodenal or hepatic artery and up to $180^{\circ}$ abutment of the SMA-as borderline resectable cases. Meta-analyses and large national cohort studies had shown higher rates of postoperative morbidity, but comparable rates of postoperative mortality and long-term overall survival after PVRs, as compared to patients without venous resections for PDAC [6,7]. In consequence, PVR was no longer considered an obstacle for curative resection if venous reconstruction was feasible. Arterial resections, mainly resections of the SMA, are still not routinely performed because of excessive morbidity and mortality rates in these patients [8]. No patient in the study cohort underwent SMA resection.

The ISGPS and NCCN [8] borderline criteria from 2014 were adopted by the current German national S3-guidelines setting the standards of pancreatic cancer treatment in Germany [9]. Consequently, German centers of pancreatic surgery are obligated to apply these mainly anatomical criteria to identify patients with borderline resectable pancreatic cancer.

As a major obstacle, the preoperative CT-based assessment of venous and arterial involvement may be inaccurate in a considerable number of patients; the sensitivity of arterial and venous invasion was demonstrated to be as low as $66 \%$ and $14 \%$ respectively [10]. Furthermore, the false positive assessment of arterial encasement occurs frequently [11]. Joo et al. recently performed a study assessing interrater reliability among radiologists evaluating resectability in PDAC [12]. The authors demonstrated an interrater reliability as low as 30\%, and in cases determined as borderline resectable, resection rates varied from $0 \%$ to $74 \%$. In consequence, the exploration of patients diagnosed as borderline resectable is currently necessary to verify inconsistent resectability determinations based on preoperative imaging.

In our study, the sensitivities of the ISGPS 2014 definition in predicting $\mathrm{R}$ status, both the UICC/AJCC R status and Leeds/Wittekind circumferential margin status, were as low as $48 \%$ and $60 \%$, respectively. Furthermore, the accuracy in predicting margin negative resection in the absence of positive ISGPS 2014 borderline resectability criteria was low, at $64 \%$ and $60 \%$, respectively.

In 2017, the International Association of Pancreatology (IAP) aimed to improve the accuracy of the borderline resectability definition by adding further biological and conditional dimensions [13]. Several studies demonstrated reduced resection rates and impaired overall survival after resection for patients with high preoperative carbohydrate antigen 19-9 (CA19-9) levels and preoperative suspicions of lymph node metastases [14-16]. The IAP 2017 borderline resectability definition introduced preoperative CA19-9 levels of more than $500 \mathrm{IU} / \mathrm{mL}$ and imaging-based suspicions of lymph node metastases as novel borderline resectability criteria. Additionally, based on a study identifying performance status as a major prognostic parameter in PDAC, a performance status of more than 2 was added as a conditional criterion of borderline resectability [17]. By expanding the definition of borderline resectability, decision making to determine resectability becomes more and more complex. In consequence, a general use of the IAP 2017 borderline definition could not be imposed internationally.

Kato et al. compared the ISGPS 2014 and NCCN guidelines to the IAP 2017 borderline definition [18]. The study disclosed that the IAP 2017 definition allowed the accurate stratification of resectable, borderline resectable and unresectable pancreatic cancers, with significantly different long-term survival estimates. In contrast, the ISGPS/NCCN-based stratification of resectability could not distinguish between different prognostic subgroups. In the current study, both the ISGPS 2014 and IAP 2017 borderline criteria qualified as prognostic parameters in the univariate analysis. However, in the multivariate analysis, only SMA stranding, but not the other borderline criteria, remained an independent prognostic factor.

In the current study, perivascular SMA stranding was evaluated as potential borderline resectability criterion. Perivascular SMA stranding in PDAC patients can be visualized in preoperative CT-based imaging. In previous studies, this phenomenon was described as cancer-infiltration-mimicking fibrous adhesion or an inflammatory reaction $[5,10,11]$. Kato et al. found impaired overall survival rates and a higher rate of early distant metastasis in patients with a reticular pattern surrounding the 
SMA [19]. We recently demonstrated that perivascular SMA stranding is a preoperative radiological marker of fibrotic stroma resection (S+ status) at the mesopancreatic resection margin [20]. In this study, $\mathrm{S}+$ status was a strong negative prognostic parameter and served as a surrogate of aggressive tumor biology and advanced tumor cell dissemination. These results are in line with the current study. SMA stranding was associated with perineural invasion and lymph node infiltration, mirroring increased local tumor invasion. It may also be speculated that perivascular SMA stranding is a morphological correlate of very pronounced perineural invasion. Perineural invasion is well-known as one of the main causes of local tumor recurrence and metastasis and was demonstrated as one of the major prognostic parameters in PDAC [21-23]. In the current study, perivascular SMA stranding qualified as an independent prognostic factor, stronger than established prognostic parameters like ASA score, $\mathrm{N}$ status, lymphovascular invasion or $\mathrm{R}$ status.

Alterations of the SMPV were assessed as a further criterion of borderline resectability in the current study. The prognostic relevance of the amount of SMPV tumor involvement, tumor localization, amount of encasement, distortion or occlusion is under debate, and recent studies present conflicting results $[4,13,24,25]$. The most probable cause of these heterogenous results is the difficulty in assessing the SMPV circumferentially in preoperative imaging. To simplify the evaluation of preoperative imaging and to improve reproducibility, we decided to assess any alterations of SMPV-such as tumor narrowing, distortion or occlusion-as a borderline resectability criterion. A current study demonstrated that any irregularity of the SMPV contour is predictive of venous involvement and also qualifies as a negative prognostic factor [26]. In our study, SMPV alterations qualified as a negative predictive parameter in the univariate analysis, but not in the multivariate analysis. The criterion of SMPV was most likely ruled out by other strong independent prognostic factors, such as SMA stranding or $\mathrm{N}$ status. However, SMPV alterations are an important component of the novel borderline resectability definition, as they considerably increase the accuracy of $\mathrm{R}$ status prediction as compared to SMA stranding alone. Combining both SMA stranding and SMPV alterations, the novel borderline resectability scoring system qualifies to safely predict $\mathrm{R} 0$ resection (group A), and to identify patients with an increased risk of R1 resection (group B) and patients with a high risk of R1 resection (group C) based on simple preoperative radiological criteria.

$\mathrm{R} 0$ resection is the major outcome parameter in borderline resectable patients, and $\mathrm{R}$ status is one of the most important prognostic parameters in pancreatic cancer patients [27-29]. However, the current study is the first, to our knowledge, evaluating the accuracy of different borderline definition criteria in predicting R status. In general, borderline resectable PDAC is defined as technically resectable with a high risk of margin positive resection. The sensitivity of ISGPS 2014 and IAP 2017 definitions in predicting R status rangedaround $50 \%$, and thus did not qualify to predict a high risk $(>50 \%)$ of margin positive resection. In contrast, the novel borderline criteria for SMA stranding and SMPV alterations together had a high sensitivity mainly in predicting Leeds/Wittekind $\mathrm{R}$ status, mirroring the actual definition of borderline resectability (increased risk of R1 resection). Furthermore, the probability of R0 resection in the absence of the novel borderline criteria was as high as $70 \%$ to $80 \%$, as compared to $30 \%$ to 50\% for the ISGPS 2014 and IAP 2017 definition criteria. Consequently, the novel borderline definition may serve as an important tool to safely achieve R0 resections.

As a major obstacle, the pathological work-up of resection margins is not standardized internationally. The UICC/AJCC criteria, mainly applied in the United States, consider resection margins positive only in case of tumor cells in direct contact with the resections margin. The Leeds/Wittekind protocol used in Europe involves a circumferential assessment of all tumor margins, and a distinction is made between "R0 narrow", with less than $1 \mathrm{~mm}$ distance between tumor cells and the resection margin, and "R0 wide", showing a minimum distance of $1 \mathrm{~mm}$ between tumor cells and the resection margin $[30,31]$. Several studies and meta-analyses have demonstrated improved overall survival rates following $\mathrm{R} 0$ wide resections as compared to $\mathrm{R} 0$ narrow and $\mathrm{R} 1$ resections $[27,32,33]$. The novel borderline definition criteria show a moderate sensitivity in predicting UICC/AJCC R status and a high sensitivity in predicting Leeds/Wittekind circumferential margin status. 
This study is limited by its retrospective nature, and potential bias or cofounders cannot be completely ruled out. To validate the results of this study, future large-scale cohort studies or prospective trials should be performed.

Mainly in borderline resectable PDAC, neoadjuvant therapy is of growing importance. Patients in the current study were not treated with neoadjuvant therapy. The German S3-guideline for the treatment of pancreatic cancer recommends upfront resection in borderline resectable PDAC patients, and neoadjuvant therapy is only allowed in the context of clinical studies [9]. However, current studies show promising results for neoadjuvant treatment in borderline resectable PDAC patients [34,35]. Multiple studies and meta-analyses demonstrated a higher R0 resection rate and improved overall survival rates in borderline resectable PDAC patients undergoing neoadjuvant therapy [36-38]. Different treatment regimens are currently assessed in randomized trials for borderline resectable PDAC patients. The ALLIANCE A021101 pilot study recently demonstrated an overall survival of 22 months and an $\mathrm{R} 0$ resection rate as high as $64 \%$ in borderline resectable PDAC patients undergoing neoadjuvant therapy with modified FOLFIRINOX and stereotactic radiotherapy [39]. The benefit of radiotherapy, as compared to FOLFIRINOX-based chemotherapy alone, remains unclear and will be further evaluated in the ALLIANCE A021501 trial [40]. However, due to the various complex definitions of borderline resectability, patient selection for neoadjuvant therapy is challenging and has not yet been standardized. The novel borderline resectability scoring system may serve to simplify patient selection for neoadjuvant therapy. Mainly patients with a considerable risk of R1 resection should undergo neoadjuvant therapy. Based on the results of the current study, we propose to consider neoadjuvant therapy in group B patients with an increased risk of R1 resection and in group C patients with a high risk of $\mathrm{R} 1$ resection, so as to improve $\mathrm{R} 0$ resection rates and, potentially, overall survival rates as well. The benefit of neoadjuvant therapy in these patient subgroups should be assessed in future studies. Furthermore, potential molecular markers may improve the accuracy of the novel borderline resectability scoring system and should be addressed in the future.

\section{Materials and Methods}

\subsection{Patients and Study Parameters}

Approval for the study was obtained from the University of Luebeck's institutional ethics committee (\#17-118A, 2017). Patients who underwent PD for PDAC were identified from a prospectively maintained database. No neoadjuvant therapy was performed. Patients with locally advanced PDAC tumors were excluded from the study. Patients included in the study underwent surgery in the period from 2013 to 2018. The following patient baseline parameters were included for the analysis: age, gender, American Society of Anesthesiologists score (ASA score) and preoperative serum levels of carbohydrate antigen 19-9 (CA19-9) in IU/mL. ASA score was dichotomized as ASA scores 0-2 versus 3-4, and serum CA19-9 was dichotomized according to the upper normal serum value of $40 \mathrm{I} \mathrm{U} / \mathrm{mL}$. The operation parameters analyzed in the study were the type of resection (Whipple, pylorus-preserving PD (PPPD), total pancreatectomy (TPE) or distal pancreatectomy), segmental portal vein resection (PVR), multivisceral resection and operation time (from incision to skin closure). Operation time was dichtomized according to the median.

Preoperative CT-based imaging was reviewed by one radiologist and two trained surgeons for the evaluation of borderline resectability criteria.

\subsection{Tests of the Diagnostic Accuracy of the Borderline Resectability Criteria}

Accuracy assessment of the borderline resectability criteria was performed following the STARD 2015 (a list of essential items for reporting diagnostic accuracy studies) [41]. For the study flow chart, see Figure 3; for list of STARD items, see Supplementary Table S1. CT scans and patients' records were assessed for borderline resectability according to the ISGPS 2014 definitions [5], and according to the IAP 2017 definitions [13], as either resectable or borderline resectable. Both definitions served 
as established reference standard tests, as the internationally established and most frequently used borderline definition criteria. According to the ISGPS 2014 definition, borderline resectable pancreatic cancer is defined as [1] no evidence of distant metastases; [2] venous involvement of the superior mesenteric vein or portal vein, with distortion or narrowing of the vein or occlusion of the vein, with suitable vessels proximal and distal, allowing for safe resection and replacement; [3] gastroduodeneal artery encasement up to the hepatic artery, with either short segment encasement or direct or indirect abutment of the hepatic artery without extension to the celiac axis; and [4] tumor abutment of the SMA not exceeding $180^{\circ}$ of the circumference of the vessel wall. According to the IAP 2017 definition, borderline resectable PDAC is defined as [1] a tumor contact angle of $180^{\circ}$ or greater, or invasion of the SMV/PV with bilateral narrowing or occlusion, but not exceeding the inferior border of the duodenum; [2] tumor contact with the SMA or celiac axis at an angle of less than $180^{\circ}$ without showing stenosis or deformity, or tumor abutment of the common hepatic artery without showing tumor contact with the proper hepatic artery and/or celiac axis; [3] clinical findings giving rise to the suspicion of distant metastases, including serum CA19-9 levels of more than $500 \mathrm{U} / \mathrm{mL}$ or regional lymph node metastasis; [4] patients with resectable PDAC, but a performance status of 2 or more [13].

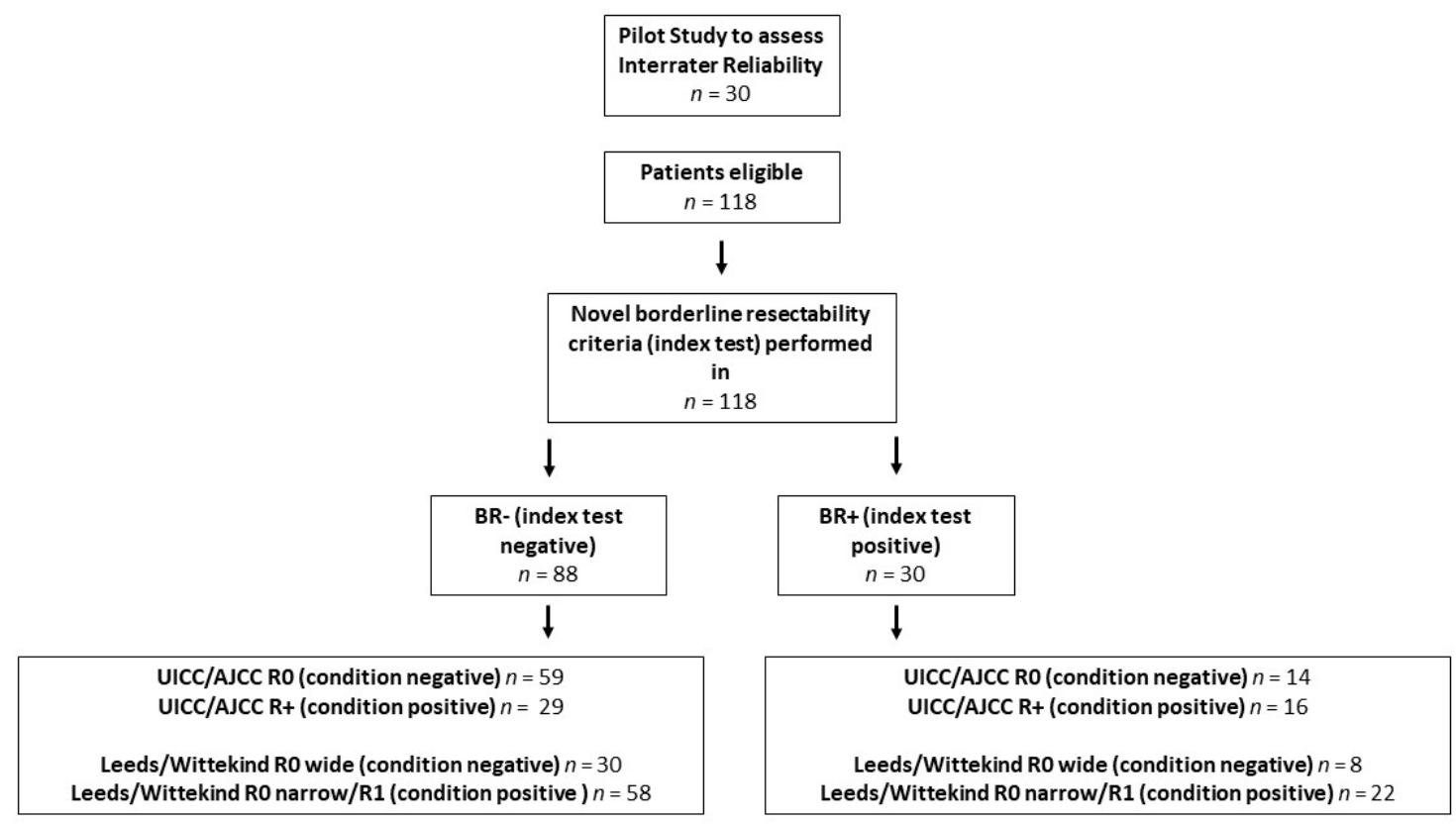

Figure 3. A Study Flow Chart according to the STARD 2015 List of essential items for reporting diagnostic accuracy studies. BR-: not borderline resectable; BR+: borderline resectable; UICC/AJCC: Union International Contre le Cancer/American Joint Committee on Cancer.

Furthermore, novel simplified borderline criteria were evaluated as an index test. Perivascular SMA stranding was considered positive in case of perivascular hazy density of the fat plane. Regarding the SMPV, any radiologically determinable vessel abnormalities such as tumor narrowing, vessel distortion or vessel occlusion were classified as SMPV alterations. The novel borderline resectability criteria are depicted in Figure 4a,b. In a pilot study of 30 patients, interrater reliability was assessed for the novel criteria. Two radiologists and two surgeons, blinded for clinical patient criteria and outcome variables, reviewed the CT-based imaging of 30 patients, assessing SMA stranding and SMPV alterations as either positive or negative. The interrater reliability for SMA stranding was $90 \%$, while the interrater reliability for SMPV alterations was $87 \%$. For the patients included in the study, preoperative CT-based imaging was reviewed by one independent radiologist and two independent surgeons blinded for clinical patient criteria and outcome variables. The evaluation of the ISGPS 2014 and IAP 2017 reference standard borderline resectability criteria-as well as of the novel radiological criteria, SMA alterations and SMPV alterations-was performed. 
a

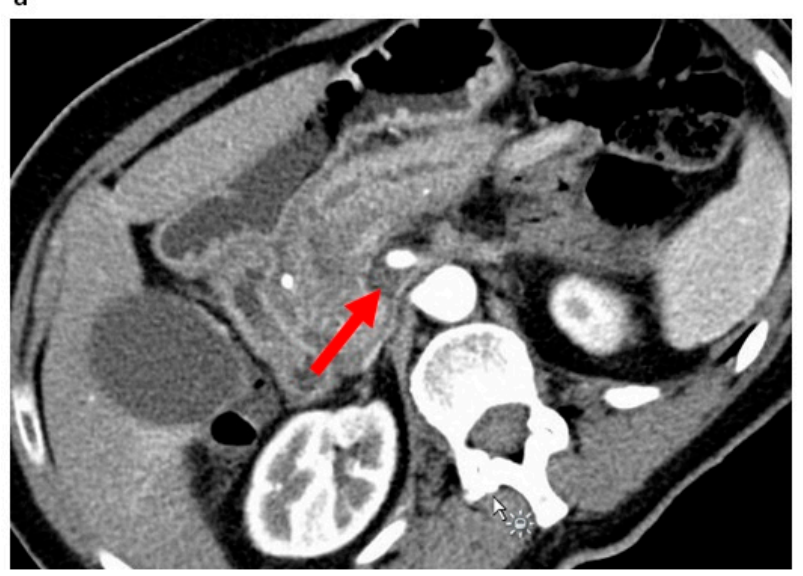

b

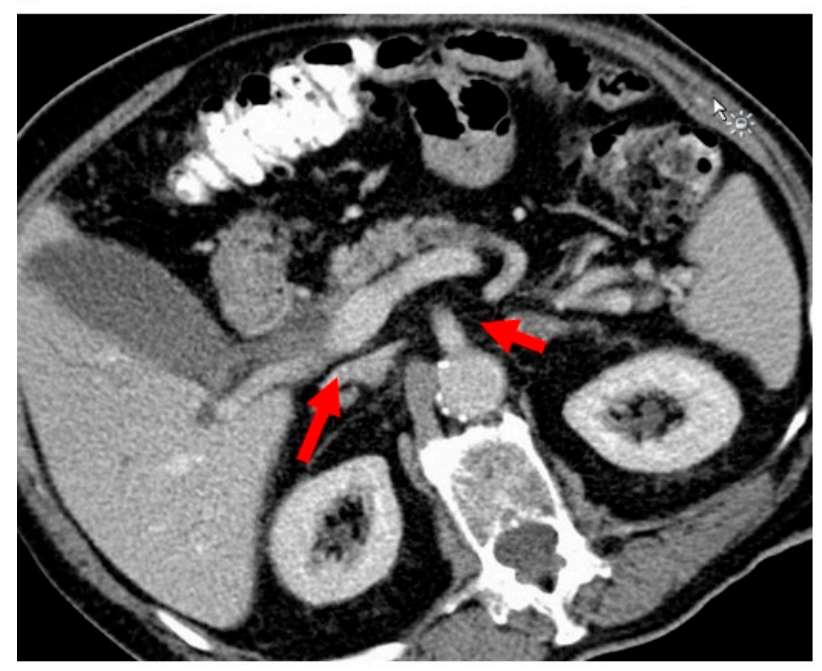

Figure 4. The novel resectability criteria: SMA stranding and superior mesenterico-portal vein alteration (SMPV) alterations. (a). Perivascular stranding of the superior mesenteric artery in a patient with pancreatic head cancer; (b) Tumor narrowing and distortion of the superior mesenteric vein and the absence of perivascular stranding of the superior mesenteric artery in a patient with pancreatic head cancer. SMA: superior mesenteric artery; SMPV: superior mesenterico-portal vein.

The histopathological parameters included in the analysis were the $\mathrm{T}$ stage, $\mathrm{N}$ stage and $\mathrm{M}$ stage grading according to Broders [42]; the lymph node ratio (LNR); lymphovascular invasion (L); vascular invasion (V) and perineural invasion (Pn). T stage was dichotomized as T1-2 versus T3-4, the LNR was dichotomized according to the median LNR value, and Broders grading was dichotomized as G1-2 versus G3-4. R status was re-evaluated by an experienced pathologist and two trained surgeons according to the UICC/AJCC criteria (conventional $\mathrm{R}$ status), as $\mathrm{R} 0$ if no tumor cells were detected at the resection margin, versus R1 if tumor cells were present right at the resection margin [43]. Specimens were further classified according to Leeds/Wittekind circumferential R status, as R1 if tumor cells were detected at the resection margin, versus R0 narrow if tumor cells were detected within $1 \mathrm{~mm}$ distance of the resection margin, versus $\mathrm{R} 0$ wide if the distance between tumor cells and resection margin was more than $1 \mathrm{~mm}[30,31]$. TNM staging was performed according to the 7th edition of the American Joint Committee on Cancer (AJCC) [44]. The postoperative parameters were reoperation and adjuvant therapy. Overall survival time was defined from surgery until death from any cause.

For estimating the diagnostic accuracy of borderline resectability definitions in predicting $\mathrm{R}$ status, cross tabulation was performed to determine the sensitivity and negative predictive value of reference standards (ISGPS 2014 and IAP 2017) and the index test (SMA stranding and SMPV alterations). 
Sensitivity is defined as the proportion of patients with a positive test result (patients defined as borderline resectable) who have a positive condition ( $\mathrm{R}+$ resection). The negative predictive value is defined as the proportion of patients with a negative test result (patients defined as NOT being borderline resectable) who have a negative condition (R0 resection).

\subsection{Statistics}

For statistical analysis, IBM SPSS Statistics for Windows, Version 25.0 was used. Continuous and categorical variables were expressed as median/range and absolute/relative frequencies, respectively. Statistical testing was performed by Chi-squared tests. Interrater reliability was calculated as the joint probability of agreement (percentage of the time the raters agree in the nominal rating system), and Fleiss' Kappa was additionally calculated to assess interrater reliability for more than four raters. Median overall survival estimates were determined with the Kaplan-Meier method, the log-rank-test and the Cox proportional hazards model. Multivariable analysis was also performed with the Cox proportional hazards model. The significance level was set to $p<0.05$ (two-sided). All confidence intervals (CI) reported are $95 \%$ confidence intervals.

\section{Conclusions}

In conclusion, the preoperative CT-based assessment of any SMPV alterations and of perivascular SMA stranding qualified as new definition criteria of borderline resectability in PDAC patients. These criteria may be applied to accurately predict $\mathrm{R}$ status.

Supplementary Materials: The following are available online at http://www.mdpi.com/2072-6694/12/4/882/s1, Table S1: STARD 2015 List of Study Items.

Author Contributions: Conceptualization, formal analysis, methodology, Writing-original draft preparation, approval for final version to be published: L.B., K.M. (Katharina Mueller), S.Z., P.B., U.F.W. Acquisition of data, writing review for important intellectual content and editing, giving final approval of the version to be published: S.S., E.P., H.L., K.C.H., S.D., D.B., T.K.; Radiological Review: K.M. (Katharina May), S.S., S.D., L.B., K.M. (Katharina Mueller), U.F.W.; Histopathological review: P.B. All authors have read and agree to the published version of the manuscript.

Funding: This research received no external funding.

Conflicts of Interest: The authors declare no conflict of interest.

\section{References}

1. Lillemoe, K.D.; Yeo, C.J.; Cameron, J.L. Pancreatic cancer: State-of-the-art care. CA Cancer J. Clin. 2000, 50, 241-268. [CrossRef] [PubMed]

2. Howlader, N.; Noone, A.; Krapcho, M.; Garshell, J.; Miller, D.; Altekruse, S. SEER Cancer Statistics Review, 1975-2012; National Cancer Institute: Bethesda, MD, USA, 2015.

3. Barugola, G.; Partelli, S.; Crippa, S.; Capelli, P.; D'Onofrio, M.; Pederzoli, P.; Falconi, M. Outcomes after resection of locally advanced or borderline resectable pancreatic cancer after neoadjuvant therapy. Am. J. Surg. 2012, 203, 132-139. [CrossRef] [PubMed]

4. Katz, M.H.; Marsh, R.; Herman, J.M.; Shi, Q.; Collison, E.; Venook, A.P.; Kindler, H.L.; Alberts, S.R.; Philip, P.; Lowy, A.M.; et al. Borderline resectable pancreatic cancer: Need for standardization and methods for optimal clinical trial design. Ann. Surg. Oncol. 2013, 20, 2787-2795. [CrossRef] [PubMed]

5. Bockhorn, M.; Uzunoglu, F.G.; Adham, M.; Imrie, C.; Milicevic, M.; Sandberg, A.A.; Asbun, H.J.; Bassi, C.; Büchler, M.; Charnley, R.M.; et al. Borderline resectable pancreatic cancer: A consensus statement by the International Study Group of Pancreatic Surgery (ISGPS). Surgery 2014, 155, 977-988. [CrossRef]

6. Zhou, Y.; Zhang, Z.; Liu, Y.; Li, B.; Xu, D. Pancreatectomy Combined with Superior Mesenteric Vein-Portal Vein Resection for Pancreatic Cancer: A Meta-analysis. World J. Surg. 2012, 36, 884-891. [CrossRef]

7. Worni, M.; Castleberry, A.W.; Clary, B.M.; Gloor, B.; Carvalho, E.; Jacobs, D.O.; Pietrobon, R.; Scarborough, J.E.; White, R.R. Concomitant Vascular Reconstruction During Pancreatectomy for Malignant Disease: A Propensity Score-Adjusted, Population-Based Trend Analysis Involving 10206 Patients. JAMA Surg. 2013, 148, 331-338. [CrossRef] 
8. Mollberg, N.; Rahbari, N.N.; Koch, M.; Hartwig, W.; Hoeger, Y.; Büchler, M.W.; Weitz, J. Arterial resection during pancreatectomy for pancreatic cancer: A systematic review and meta-analysis. Ann. Surg. 2011, 254, 882-893. [CrossRef]

9. Seufferlein, T.; Porzner, M.; Becker, T.; Budach, V.; Ceyhan, G.; Esposito, I.; Fietkau, R.; Follmann, M.; Friess, H.; Galle, P.; et al. S3-guideline exocrine pancreatic cancer. Z. Gastroenterol. 2013, 51, 1395-1440.

10. Li, H.; Zeng, M.S.; Zhou, K.R.; Jin, D.Y.; Lou, W.H. Pancreatic adenocarcinoma: The different CT criteria for peripancreatic major arterial and venous invasion. J. Comput. Assist. Tomogr. 2005, 29, 170-175. [CrossRef]

11. Nakayama, Y.; Yamashita, Y.; Kadota, M.; Takahashi, M.; Kanemitsu, K.; Hiraoka, T.; Hirota, M.; Ogawa, M.; Takeya, M. Vascular encasement by pancreatic cancer: Correlation of CT findings with surgical and pathologic results. J. Comput. Assist. Tomogr. 2001, 25, 337-342. [CrossRef]

12. Joo, I.; Lee, J.M.; Lee, E.S.; Son, J.-Y.; Lee, D.H.; Ahn, S.J.; Chang, W.; Lee, S.M.; Kang, H.J.; Yang, H.K. Preoperative CT Classification of the Resectability of Pancreatic Cancer: Interobserver Agreement. Radiology 2019, 293, 343-349. [CrossRef] [PubMed]

13. Isaji, S.; Mizuno, S.; Windsor, J.A.; Bassi, C.; Fernández-del Castillo, C.; Hackert, T.; Hayasaki, A.; Katz, M.H.G.; Kim, S.W.; Kishiwada, M.; et al. International consensus on definition and criteria of borderline resectable pancreatic ductal adenocarcinoma 2017. Pancreatology 2018, 18, 2-11. [CrossRef] [PubMed]

14. Ferrone, C.R.; Finkelstein, D.M.; Thayer, S.P.; Muzikansky, A.; Fernandez-del Castillo, C.; Warshaw, A.L. Perioperative CA19-9 levels can predict stage and survival in patients with resectable pancreatic adenocarcinoma. J. Clin. Oncol. 2006, 24, 2897-2902. [CrossRef] [PubMed]

15. Barton, J.G.; Bois, J.P.; Sarr, M.G.; Wood, C.M.; Qin, R.; Thomsen, K.M.; Kendrick, M.L.; Farnell, M.B. Predictive and prognostic value of CA 19-9 in resected pancreatic adenocarcinoma. J. Gastrointest. Surg. 2009, 13, 2050-2058. [CrossRef] [PubMed]

16. Hartwig, W.; Strobel, O.; Hinz, U.; Fritz, S.; Hackert, T.; Roth, C.; Büchler, M.W.; Werner, J. CA19-9 in potentially resectable pancreatic cancer: Perspective to adjust surgical and perioperative therapy. Ann. Surg. Oncol. 2013, 20, 2188-2196. [CrossRef] [PubMed]

17. Tas, F.; Sen, F.; Odabas, H.; Kilic, L.; Keskin, S.; Yildiz, I. Performance status of patients is the major prognostic factor at all stages of pancreatic cancer. Int. J. Clin. Oncol. 2013, 18, 839-846. [CrossRef]

18. Kato, Y.; Yamada, S.; Tashiro, M.; Sonohara, F.; Takami, H.; Hayashi, M.; Kanda, M.; Kobayashi, D.; Tanaka, C.; Nakayama, G.; et al. Biological and conditional factors should be included when defining criteria for resectability for patients with pancreatic cancer. HPB (Oxford) 2019, 21, 1211-1218. [CrossRef]

19. Kato, T.; Ban, D.; Tateishi, U.; Ogura, T.; Ogawa, K.; Ono, H.; Mitsunori, Y.; Kudo, A.; Tanaka, S.; Tanabe, M. Reticular pattern around superior mesenteric artery in computed tomography imaging predicting poor prognosis of pancreatic head cancer. J. Hepatobiliary Pancreat. Sci. 2020, 27, 114-123. [CrossRef]

20. Wellner, U.F.; Krauss, T.; Csanadi, A.; Lapshyn, H.; Bolm, L.; Timme, S.; Kulemann, B.; Hoeppner, J.; Kuesters, S.; Seifert, G.; et al. Mesopancreatic stromal clearance defines curative resection of pancreatic head cancer and can be predicted preoperatively by radiologic parameters: A retrospective study. Medicine 2016, 95, e2529. [CrossRef]

21. Gasparini, G.; Pellegatta, M.; Crippa, S.; Lena, M.S.; Belfiori, G.; Doglioni, C.; Taveggia, C.; Falconi, M. Nerves and Pancreatic Cancer: New Insights into a Dangerous Relationship. Cancers 2019, 11, 893. [CrossRef]

22. Tanaka, M.; Mihaljevic, A.L.; Probst, P.; Heckler, M.; Klaiber, U.; Heger, U.; Büchler, M.W.; Hackert, T. Meta-analysis of recurrence pattern after resection for pancreatic cancer. Br. J. Surg. 2019, 106, 1590-1601. [CrossRef] [PubMed]

23. Liang, D.; Shi, S.; Xu, J.; Zhang, B.; Qin, Y.; Ji, S.; Xu, W.; Liu, J.; Liu, L.; Liu, C.; et al. New insights into perineural invasion of pancreatic cancer: More than pain. Biochim. Biophys. Acta 2016, 1865, 111-122. [CrossRef] [PubMed]

24. Nakao, A.; Kanzaki, A.; Fujii, T.; Kodera, Y.; Yamada, S.; Sugimoto, H.; Nomoto, S.; Nakamura, S.; Morita, S.; Takeda, S. Correlation between radiographic classification and pathological grade of portal vein wall invasion in pancreatic head cancer. Ann. Surg. 2012, 255, 103-108. [CrossRef] [PubMed]

25. Hirono, S.; Kawai, M.; Okada, K.; Miyazawa, M.; Shimizu, A.; Kitahata, Y.; Ueno, M.; Yamaue, H. Pancreatic neck cancer has specific and oncologic characteristics regarding portal vein invasion and lymph node metastasis. Surgery 2015, 159, 426-440. [CrossRef] [PubMed] 
26. Kaissis, G.A.; Lohofer, F.K.; Ziegelmayer, S.; Danner, J.; Jager, C.; Schirren, R.; Ankerst, D.; Ceyhan, G.O.; Friess, H.; Rummeny, E.J.; et al. Borderline-resectable pancreatic adenocarcinoma: Contour irregularity of the venous confluence in pre-operative computed tomography predicts histopathological infiltration. PLoS ONE 2019, 14, e0208717. [CrossRef]

27. Niesen, W.; Hank, T.; Buchler, M.; Strobel, O. Local radicality and survival outcome of pancreatic cancer surgery. Ann. Gastroenterol. Surg. 2019, 3, 464-475. [CrossRef]

28. Butturini, G.; Stocken, D.D.; Wente, M.N.; Jeekel, H.; Klinkenbijl, J.H.; Bakkevold, K.E.; Takada, T.; Amano, H.; Dervenis, C.; Bassi, C.; et al. Influence of resection margins and treatment on survival in patients with pancreatic cancer: Meta-analysis of randomized controlled trials. Arch. Surg. 2008, 143, 75-83. [CrossRef]

29. Wellner, U.F.; Makowiec, F.; Bausch, D.; Höppner, J.; Sick, O.; Hopt, U.T.; Keck, T. Locally advanced pancreatic head cancer: Margin-positive resection or bypass? ISRN Surg. 2012, 2012, 513241. [CrossRef]

30. Menon, K.V.; Gomez, D.; Smith, A.M.; Anthoney, A.; Verbeke, C.S. Impact of margin status on survival following pancreatoduodenectomy for cancer: The Leeds Pathology Protocol (LEEPP). HPB (Oxford) 2009, 11, 18-24. [CrossRef]

31. Wittekind, C.; Compton, C.; Quirke, P.; Nagtegaal, I.; Merkel, S.; Hermanek, P.; Sobin, L.H. A uniform residual tumor $(\mathrm{R})$ classification: Integration of the $\mathrm{R}$ classification and the circumferential margin status. Cancer 2009, 115, 3483-3488. [CrossRef]

32. Chandrasegaram, M.D.; Goldstein, D.; Simes, J.; Gebski, V.; Kench, J.G.; Gill, A.J.; Samra, J.S.; Merrett, N.D.; Richardson, A.J.; Barbour, A.P. Meta-analysis of radical resection rates and margin assessment in pancreatic cancer. Br. J. Surg. 2015, 102, 1459-1472. [CrossRef] [PubMed]

33. Kim, K.S.; Kwon, J.; Kim, K.; Chie, E.K. Impact of Resection Margin Distance on Survival of Pancreatic Cancer: A Systematic Review and Meta-Analysis. Cancer Res. Treat. 2017, 49, 824-833. [CrossRef] [PubMed]

34. Silvestris, N.; Brunetti, O.; Vasile, E.; Cellini, F.; Cataldo, I.; Pusceddu, V.; Cattaneo, M.; Partelli, S.; Scartozzi, M.; Aprile, G.; et al. Multimodal treatment of resectable pancreatic ductal adenocarcinoma. Crit. Rev. Oncol. Hematol. 2017, 111, 152-165. [CrossRef] [PubMed]

35. Silvestris, N.; Longo, V.; Cellini, F.; Reni, M.; Bittoni, A.; Cataldo, I.; Partelli, S.; Falcini, M.; Scarpa, A.; Brunetti, O.; et al. Neoadjuvant multimodal treatment of pancreatic ductal adenocarcinoma. Crit. Rev. Oncol. Hematol. 2016, 98, 309-324. [CrossRef] [PubMed]

36. Lee, J.H.; Kang, C.M.; Bang, S.M.; Choi, J.Y.; Seong, J.S.; Hwang, H.K.; Choi, S.H.; Lee, W.J. The role of neoadjuvant chemoradiation therapy in patients with borderline resectable pancreatic cancer with isolated venous vascular involvement. Medicine 2015, 94, e1233. [CrossRef] [PubMed]

37. Katz, M.H.; Fleming, J.B.; Bhosale, P.; Varadhachary, G.; Lee, J.E.; Wolff, R.; Wang, H.; Abbruzzese, J.; Pisters, P.W.; Vauthey, N.J.; et al. Response of borderline resectable pancreatic cancer to neoadjuvant therapy is not reflected by radiographic indicators. Cancer 2012, 118, 5749-5756. [CrossRef] [PubMed]

38. Boone, B.A.; Steve, J.; Krasinskas, A.M.; Zureikat, A.H.; Lembersky, B.C.; Gibson, M.K.; Stoller, R.G.; Zeh, H.J.; Bahary, N. Outcomes with FOLFIRINOX for borderline resectable and locally unresectable pancreatic cancer. J. Surg. Oncol. 2013, 108, 236-241. [CrossRef]

39. Katz, M.H.G.; Shi, Q.; Ahmad, S.A.; Herman, J.M.; Marsh R de, W.; Collisson, E.; Schwartz, L.; Frankel, W.; Martin, R.; Conway, W.; et al. Preoperative Modified FOLFIRINOX Treatment Followed by Capecitabine-Based Chemoradiation for Borderline Resectable Pancreatic Cancer: Alliance for Clinical Trials in Oncology Trial A021101. JAMA Surg. 2016, 151, e161137. [CrossRef]

40. Katz, M.H.G.; Ou, F.-S.; Herman, J.M.; Ahmad, S.A.; Wolpin, B.; Marsh, R.; Behr, S.; Shi, Q.; Chuong, M.; Schwartz, L.H.; et al. Alliance for clinical trials in oncology (ALLIANCE) trial A021501: Preoperative extended chemotherapy vs. chemotherapy plus hypofractionated radiation therapy for borderline resectable adenocarcinoma of the head of the pancreas. BMC Cancer 2017, 17, 505. [CrossRef]

41. Bossuyt, P.M.; Reitsma, J.B.; Bruns, D.E.; Gatsonis, C.A.; Glasziou, P.P.; Irwig, L.; Lijmer, J.G.; Moher, D.; Rennie, D.; de Vet, H.C.; et al. STARD 2015: An updated list of essential items for reporting diagnostic accuracy studies. BMJ 2015, 351, h5527. [CrossRef]

42. Broders, A.C., Sr. Malignant neoplasia of normally situated and heterotopic lymphoid tissue and its numerical microscopic grading. Tex. State J. Med. 1953, 49, 234. [PubMed] 
43. Raut, C.P.; Tseng, J.F.; Sun, C.C.; Wang, H.; Wolff, R.A.; Crane, C.H.; Hwang, R.; Vauthey, J.N.; Abdalla, E.K.; Lee, J.E.; et al. Impact of resection status on pattern of failure and survival after pancreaticoduodenectomy for pancreatic adenocarcinoma. Ann. Surg. 2007, 246, 52-60. [CrossRef] [PubMed]

44. Edge, S.B.; Compton, C.C. The American Joint Committee on Cancer: The 7th edition of the AJCC cancer staging manual and the future of TNM. Ann. Surg. Oncol. 2010, 17, 1471-1474. [CrossRef] [PubMed]

(C) 2020 by the authors. Licensee MDPI, Basel, Switzerland. This article is an open access article distributed under the terms and conditions of the Creative Commons Attribution (CC BY) license (http://creativecommons.org/licenses/by/4.0/). 